\title{
Der Fe/P-Quotient des Sediments als Merkmal des Stoffumsatzes in Brackwässern
}

\author{
JÜRGEN OVERBECK \\ Hydrobiologische Anstalt der Max-Planck-Gesellschaft, Plön, Holstein
}

\begin{abstract}
The Fe/P-quotient of mud as an indicator of the turn-over in brackish waters. The relation between phosphate and iron is of great importance in assessing the phosphate content of natural waters, since $\mathrm{Fe}(\mathrm{OH})_{3}$, which is formed in the presence of oxygen, binds phosphate and fixes it in the sediment. Through exchange of ferric hydroxide with iron sulphide by means of hydrogen sulphide, the adsorptive linkage between iron and phosphorus is broken, and the phosphate ions will go badk into solution. The sulphate content of the natural waters and the bacterial sulphate reduction connected with it are therefore of great importance for the phosphate content. In marine and brackish waters liberation of phosphate reaches extremely high intensities because of the high sulphate content. This situation was studied in the brackish waters of the Isle of Rügen (Baltic Sea) by means of sediment analyses. More than 70 soil profiles were collected and analyzed with respect to organic substances, iron and phosphate content. Considering the relationships between these three factors, it could be shown that in sediments containing less than $5 \%$ organic substances, the iron and phosphorus content is lower, and the iron/phosphorus quotient is about 12. With rising concentrations of organic substances, the iron and phosphorus content increases, iron increasing fourfold and phosphorus only doubling. Thus the iron-phosphorus quotient reaches an average of 23 . Only in highly productive brackish water areas does the $\mathrm{Fe} / \mathrm{P}$ quotient attain such a high value. An extensive production of organic material, an effective phosphate mobilization and a high $\mathrm{Fe} / \mathrm{P}$ quotient are thus causally combined.
\end{abstract}

\section{EINLEITUNG}

Nachdem in einer früheren Mitteilung (OVERBECK 1962) der überragende Anteil des Nannoplanktons an der Planktonproduktion der Rügenschen Brackwässer dargestellt wurde, soll in der vorliegenden Arbeit der Einfluß des Sediments auf die Produktionshöhe untersucht werden. Der Austausch zwischen Schlamm und Wasser hat bekanntlich eine entscheidende Bedeutung für die Nährstoffversorgung eines Gewässers. Die Bakterienmassen des Sediments führen die aeroben und anaeroben Umsetzungen durch, die schließlich die Mineralisierung der geformten organischen Substanz verursachen. Aus dem Sediment werden die aus organischer Bindung befreiten Mineralien durch den Schlamm-Wasseraustausch erneut dem Wasser und damit der pflanzlichen Produktion zugeführt. Der Schlamm ist hierbei das riesige Reservoir, aus dem die Nährstoffe in das Wasser gelangen. E in Mechanismus für diesen Austausch - speziell für die Abgabe von Phosphat aus dem Schlamm - ist bekanntlich von ganz besonderer 
Wichtigkeit, nämlich das Eisen-Phosphor-Verhältnis. Auf die Verkettung des Phosphathaushalts mit dem des Eisens haben zuerst Einsele (1936) und OHLE (1937) hingewiesen. Nach diesen Untersuchungen scheidet $\mathrm{Fe}\left(\mathrm{HCO}_{3}\right)_{2}$-haltiges Wasser bei Anwesenheit von Sauerstoff Eisen als $\mathrm{Fe}(\mathrm{OH})_{3}$ ab. Im Wasser gelöstes Phosphat wird dabei unter adsorptiver Bindung an $\mathrm{Fe}(\mathrm{OH})_{3}$ aus dem Phosphatkreislauf ausgeschieden und im Sediment festgelegt (ErNsele 1936). Die Adsorption des $\mathrm{PO}_{4}$-Anions an das kolloidale $\mathrm{Fe}(\mathrm{OH})_{3}$ ist $\mathrm{pH}$-abhängig und ist im alkalischen Bereich stark zurückgedrängt (Ohle 1937). Auch werden die Prozesse unter anderem dadurch kompliziert, daß in kalkarmen Braunwasserseen die dort reich vorhandenen Huminsäureverbindungen Eisen adsorbieren, wodurch eine frühzeitige Ausfällung des Eisenhydroxyds verhindert wird (OHLE 1935).

Widhtig ist nun, daß die Eisen-Phosphor-Beziehung durch den Sulfat- bzw. $\mathrm{H}_{2} \mathrm{~S}$ Gehalt des Gewässers oder des Schlammes entscheidend beeinflußt wird: Die bakterielle Sulfatreduktion nimmt eine beherrschende Stellung im Sediment ein. Durch den entstandenen Schwefelwasserstoff wird das Eisen als Sulfid ausgefällt oder bei aeroben Verhältnissen im Schlamm dazu umgewandelt. Dadurch wird die Phosphatfestlegung durch Ferrihydroxyd aufgehoben, die Phosphationen bleiben in Lösung oder gelangen aus dem Sediment in das Wasser. Die Bedeutung des Sulfats für diese Phosphatmobilisierung ist gar nicht hoch genug einzuschätzen, und mit vollem Recht wurde Sulfat daher als „Katalysator" des limnischen Stoffkreislaufs bezeichnet (OHLE 1954a). Wenn der Schlamm eine so große Reduktionskapazität besitzt, daß er auch durch Kontakt mit sauerstoffhaltigem Wasser nicht oxydiert wird, erfolgt überhaupt keine Phosphatfestlegung mehr oder doch nur insoweit, als die Phosphationen in Organismen gebunden werden. Infolge der stürmisch verlaufenden bakteriellen Umsetzungen im Sediment wird auch dieser Phosphatanteil schnell wieder mineralisiert. „Ein See, der diesen Zustand erreicht hat, ist wahrhaft als eutroph zu bezeichnen" (OHLE 1953). Für Meerwasser und Brackwasser hat dieser Mechanismus infolge des im Vergleich zum Süßwasser erheblich erhöhten Sulfatgehalts (Verhältnis etwa 1:100) zweifellos eine ganz besondere Bedeutung. Auf dieser Tatsache beruhen ja die Schwierigkeiten der Abwasserbeseitigung küstennaher Großstädte durch eine einfache Einleitung ohne vorherige Aufbereitung in Meeresbuchten (OHLE 1954b).

Der Zweck der vorliegenden Untersuchung ist es, diese Verhältnisse in den Rügenschen Brackwässern zu erörtern und speziell durch Sedimentanalysen einen Einblick in die Phosphat-Eisen-Verkettung im Brackwasser zu vermitteln. Denn die durch hohen Sulfatgehalt des Wassers gesteigerte Phospharmobilisierung mußte für den Phosphatgehalt dieses Brackwassergebietes und damit für die Höhe der Planktonproduktion von entscheidender Bedeutung sein.

\section{UNTERSUCHUNGSMETHODEN}

Für die Sedimententnahme verwendeten wir einen selbstkonstruierten Bodenstecher mit Gestänge und auswechselbarem Glasrohr (Overbeck 1957). Der Bodenstecher besitzt eine Metallhülse mit scharfer Schneide, die mit Hilfe des Gestänges auch bei hartem Sandboden eine Probenentnahme gestattet. Hiermit wurden im Mai, Juli, 
September und Dezember 1954 sowie im April und Oktober 1955 über 70 Bodenprofile entnommen und nach Zerteilung in $1 \mathrm{~cm}$ lange Abschnitte (ca, 300 Proben) auf ihren Wassergehalt, Gehalt an organischer Substanz (Glühverlust), Stickstoff, GesamtPhosphat und Eisen untersucht. Im einzelnen wurde wie folgt verfahren: Zur Bestimmung des W a s e rgehaltes ließen wir eine eingewogene Menge Feuchtschlamm (ca. $5 \mathrm{~g}) 2 \times 24$ Stunden bei $100^{\circ} \mathrm{C}$ im Porzellantiegel trocknen und verwogen bis zur Gewichtskonstanz. Zur Bestimmung des Glü h v e $\mathrm{rlu}$ s t e s wurden die Proben 2 Stunden bei $600^{\circ} \mathrm{C}$ geglüht und nach dem Abkühlen im Exsikkator bis zur Gewichtskonstanz verwogen. Bei $600^{\circ} \mathrm{C}$ beginnt zwar bereits die Zersetzung von $\mathrm{CaCO}_{3}\left(\mathrm{UN}^{-}\right.$ GEMACH 1960), da aber die Sedimente kalkarm sind, dürfte dieser Fehler nicht ins Gewicht fallen. Der G e s a $\mathrm{m} \mathrm{t}-\mathrm{St}$ i cks t of $\mathrm{f}$ wurde in üblicher Weise nach dem Kjeldahl-Verfahren bestimmt. $100 \mathrm{mg}$ des getrockneten, pulverisierten und gesiebten $(0,5 \mathrm{~mm})$ Sediments wurden unter Zusatz des Selenreaktionsgemisches nach WIENINGER (Merck Nr. 8030) und $2 \mathrm{ml}$ konzentrierter Schwefelsäure in einem Kjeldahlkolben auf dem Sandbad aufgeschlossen, dann das Ammoniak in einer Mikroapparatur abdestilliert und in der Vorlage titrimetrisch bestimmt. Der Gesamt-Phosphor wurde in den meisten Fällen vergleichend nach zwei Verfahren bestimmt: nach der von OHLE (1938) angegebenen Methodik und nach einem von THomas (1955) veröffentlichten Bestimmungsverfahren. Bei dem Verfahren nach OHLE wird das luftrodkene Sediment mit konzentrierter Schwefelsäure aufgeschlossen, bei der Bestimmung nach THomas abweichend hiervon 2 Stunden in 10\% iger $\mathrm{HCl}$ auf dem Sandbad gekocht. $\mathrm{Da}$ die Bestimmungsmethodik für unsere Ergebnisse prinzipielle Bedeutung besitzt, seien beide Verfahren hier dargestellt.

\section{Gesamtphosphorbestimmung mit Scbwefelsäureaufschluß}

$50 \mathrm{mg}$ des luftrockenen, pulverisierten Sediments wurden in den von OHLE beschriebenen Glasschiffchen quantitativ in einen $100 \mathrm{ml}$ Erlenmeyerkolben gebracht, mit $0,4 \mathrm{ml} \mathrm{H}_{2} \mathrm{SO}_{4}$ conc. und $10 \mathrm{ml}$ Aqua destillata versetzt und auf dem Sandbad bis zum Auftreten von $\mathrm{SO}_{3}$-Dämpfen erhitzt. Nach dem Abkühlen gaben wir unter leichtem Erwärmen $2 \times 5$ Tropfen Perhydrol (bei kohlenstoffreichem Sediment mehr) zum Aufschluß, wodurch der Kohlenstoff vollständig oxydiert und die Schmelze farblos wird. Dann wurde wie vorgeschrieben verfahren (OHLE 1938: Neutralisieren mit Ammoniak 2,5\%, Zusatz von $2 \mathrm{ml} n$ Schwefelsäure, Zentrifugieren, Zusatz von $1 \mathrm{ml}$ 0,01\%iger KCN-Lösung). Bei vorschriftsmäßiger Durchführung des Bestimmungsganges erhält man ausgezeichnet reproduzierbare Werte. Die Notwendigkeit, neben dem Schwefelsäureaufschluß auch einen Salzsäureaufschluß zu verwenden, ergab sich daraus, daß TRAmMs (1941) mit einem Salzsäureaufschluß im Sediment des Großen Jasmunder Boddens erheblich höhere Phosphatwerte als wir ermittelte. Es war daher auch ein Salzsäureaufschluß zu überprüfen. Unsere Ergebnisse zeigen jedoch, daß beide Aufschlußverfahren recht gut übereinstimmen und die TraHmsschen Werte als zu hoch anzusehen sind. Wahrscheinlich ist der hohe Phosphatgehalt bei TRAHMs auf die unzulässige, getrennte Zugabe von Ammoniummolybdat und Schwefelsäure zurückzuführen, wodurch die Bildung von Silicomolybdänblau gefördert wird. 


\section{Gesamtphosphorbestimmung mit Salzsäureaufscbluß}

Beim Salzsäureaufschluß werden $500 \mathrm{mg}$ des trockenen Sediments im Kjeldahl-

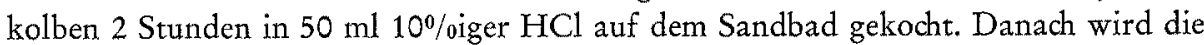
Probe in einen 100-ml-Meßkolben filtriert, auf Marke aufgefüllt und 1:10 verdünnt $(10$ auf $100 \mathrm{ml}$ ). Von dieser 2. Verdünnungsstufe werden $10 \mathrm{ml}$ entnommen und mit Paranitrophenol $(0,2 \% \mathrm{og}, 2$ Tropfen) als Indikator mit Ammoniak $(2,5 \%)$ bis zum Neutralpunkt titriert. Die neutralisierte Lösung wird in einen $100-\mathrm{ml}-\mathrm{Meßkolben}$ gespült, nach Zugabe von $1 \mathrm{ml}$ Ammonmolybdat-Schwefelsäure und $0,12 \mathrm{ml} \mathrm{SnCl}$ (nach OHLE) auf Marke aufgefüllt und nach genau 10 Minuten im Pulfrichphotometer wie beim Schwefelsäureaufschluß gemessen (15 cm Absorptionsrohr, Filter S 43). Aus der 2. Verdünnungsstufe des Salzsäureaufschlusses wurden $25 \mathrm{ml}$ entnommen und unter Zusatz von 3 Tropfen Perhydrol mit $1,3 \mathrm{ml} \mathrm{HCl}$ conc. auf ungefähr $20 \mathrm{ml}$ eingedampt, wobei $\mathrm{E}$ is en in die dreiwertige Stufe überführt wird. Nach dem Erkalten wird die Probe in einen 25-ml-Meßkolben überführt und unter Zusatz von 2,5 $\mathrm{ml}$ 50\% \% iger Ammonrhodanidlösung auf Marke aufgefüllt und nach 5 Minuten im Pulfrichphotometer in der $5 \mathrm{~cm}$ Küvette gemessen (MüLIER 1954).

\section{METHODISCHE UNTERSUCHUNGEN ZUR GESAMTPHOSPHOR- BESTIMMUNG IM BRACKWASSERSEDIMENT}

Zusammenfassend sei über einige methodische Untersuchungen berichtet, die zur Sicherung der Ergebnisse in großen Reihen durchgeführt wurden.

\section{Vergleich verschiedener Aufschlußmittel}

Um die Wirksamkeit verschiedener Aufschlußverfahren zu ermitteln, wurden Sedimentproben aus dem Breeger Bodden (Abb. 1) vergleichend mit konzentrierter Salzsäure bzw. Aqua destillata aufgeschlossen. Mit dem Aqua destillata-Aufschluß wurde $82 \%$ des Phosphors im Verhältnis zum Salzsäureaufschluß erhalten. Eine Versuchsreihe mit Königswasser, $\mathrm{HCl}$ conc., $\mathrm{H}_{2} \mathrm{SO}_{4}$ conc. und Aqua destillata ergab in der Reihenfolge der Aufschlußmittel einen Phosphorgehalt von 100\%, 96\%, 95\% bzw. 91\%. Die Untersuchungen zeigen also, daß nur ein ganz geringer Anteil des Phosphats festgelegt ist und der Hauptteil in wasserlöslicher Form vorliegt. Dieses Ergebnis ist von großer Wichtigkeit, beweist es doch die postulierte Phosphatmobilität des Brackwassersediments, die Gegenstand der vorliegenden Untersuchung ist. Der größte Teil des Eisens liegt im Sediment als Sulfid vor (tiefschwarzes Sediment, das nach Zugabe von Säure penetrant nach $\mathrm{H}_{2} \mathrm{~S}$ riecht), Phosphat wird also nicht adsorbiert. Hinzu kommt, daß in dem alkalischen Schlamm ( $\mathrm{pH}$ etwa 7,5-8,5) die Adsorption der $\mathrm{PO}_{4}$-Anionen an eventuell vorhandene andere kolloidale Träger erheblich zurückgedrängt sein muß, da organische Kolloide, um die es sich im wesentlichen handeln dürfte, im alkalischen Bereich negativ geladen sind (OHLE 1937). Auch wird die Mineralisierung in dem organisch reichen Flachwassersediment sehr rasch ablaufen. 
Ferner wissen wir aus den Untersuchungen der letzten Jahre, daß die postmortale Abgabe von Phosphat aus pflanzlichen und tierischen Zellen außerordentlich hoch ist. Nadh Krause (1961) erreicht sie beispielsweise bei Zooplankton im Extremfall $95 \%$ in 24 Stunden, das heißt $95 \%$ des in den Zellen gebundenen Phosphats ist bereits nach einem Tag frei verfügbar. Die Summe aller Faktoren bewirkt, daß das Phosphat größtenteils in wasserlöslicher, nicht gebundener Form im Brackwassersediment vorliegt. Es ist also wirklich mobilisiert.

\section{Einfuß von $\mathrm{NaCl}$ auf die Phosphatbestimmung}

Da wir es mit einem Brackwassersediment zu tun haben, war es notwendig, den Einfluß des Salzgehalts auf die Gesamtphosphorbestimmung zu untersuchen. Das Ergebnis ist aus folgenden Daten zu ersehen:

\begin{tabular}{lcc}
\multicolumn{1}{c}{ eingewogen } & Salzgebalt & gefunden \\
\hline $10 \gamma$ P pro & Aqua destillata \\
$10 \mathrm{ml}$ destilliertes Wasser & ohne NaCl \\
& $+1 \% \mathrm{NaCl}$ & $10,1 \gamma$ \\
& $+2 \% \mathrm{NaCl}$ & $10,1 \gamma$ \\
& $+3 \% \mathrm{NaCl}$ & $9,4 \gamma$ \\
& $8,8 \gamma$
\end{tabular}

Man sieht, daß mit zunehmendem Salzgehalt die Phosphatreaktion nicht unerheblich gehemmt wird. $\mathrm{Da}$ aber der Salzgehalt im Untersuchungsgebiet stets unter $1 \%$ liegt, kann man diese Salzhemmung vernachlässigen. Der Einfluß des Salzgehalts auf die Bestimmung des anorganischen Phosphats ist aus den folgenden Analysendaten zu erkennen:

\begin{tabular}{lcc}
\multicolumn{1}{c}{ eingewogen } & Salzgebalt & gefunden \\
\hline $30 \gamma$ P pro & Aqua destillata \\
11 destilliertes Wasser & ohne NaCl \\
& $+1 \% \mathrm{NaCl}$ & $29,6 \gamma$ \\
& $+2 \% \mathrm{NaCl}$ & $29,6 \gamma$ \\
& $+3 \% \mathrm{NaCl}$ & $28,2 \gamma$ \\
& $27,8 \gamma$
\end{tabular}

Der Faktor für den „Salzfehler" beträgt bei $3 \% \mathrm{NaCl} 1,07$ und entspricht damit vollständig den von Cooper (1938/39) zusammengestellten Werten für den „salt errorc. Auch hier gilt, daß die Phosphatbestimmung durch den niedrigen Salzgehalt des Standorts nicht beeinflußt wird. Dagegen wird in Ubereinstimmung mit KaLIE (1935) eine deutliche Hemmung der Phosphatreaktion durch Kupfersulfat bewirkt. So ergab ein Zusatz von $\mathrm{n} / 1000 \mathrm{CuSO}_{4}$ eine Schwächung der Farbintensität um $12 \%$. 


\section{ÜBERSICHT ÜBER DIE RÜGENSCHEN BRACKWASSER}

Abbildung 1 gibt einen Überblick über das Untersuchungsgebiet. Die Rügenschen Bodden sind flache, mit der Ostsee kommunizierende Wasserbecken, die sich in langer Kette bis weit in das Innere der Insel Rügen hinein erstrecken. Da trennende Untiefen fehlen, bilden sie ein zusammenhängendes Gewässersystem, dessen Durchschnittstiefe etwa 2,5 m beträgt. Tiefere Becken sind vor allem im Großen Jasmunder Bodden

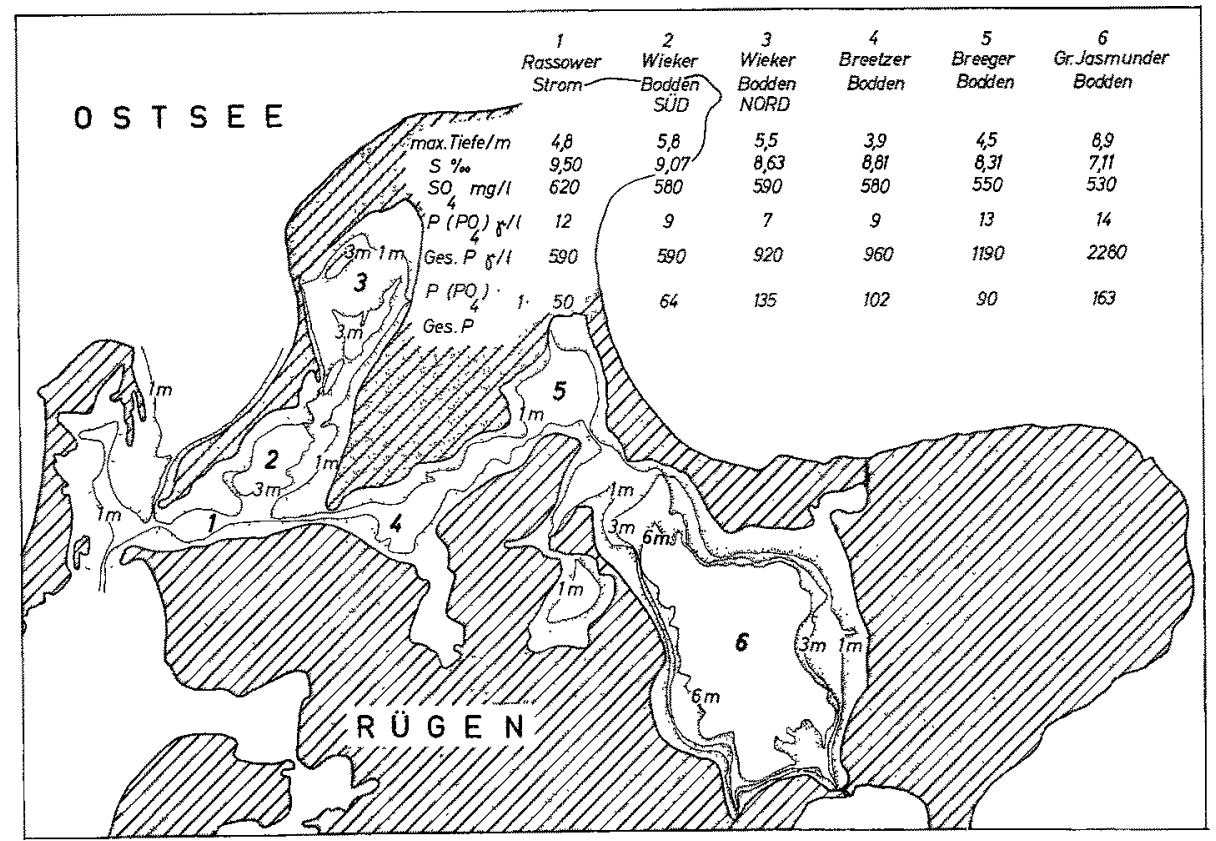

Abb. 1: Ubersicht über das Untersuchungsgebiet. Die Zahlen 1 bis 6 bezeichnen die Ortlichkeiten, an welchen die Proben entnommen wurden. Die rechts oben aufgeführten Daten repräsentieren Mittelwerte aus allen Tiefenstufen (1-m-Intervalle) im September 1957

(Maximaltiefe etwa 8 bis $9 \mathrm{~m}$ ) und in kleinerem Umfange auch im Wieker Bodden vorhanden. Die geringe Tiefe und die windbedingte Turbulenz verhindern die Ausbildung einer Sprungschicht. Die Gewässer sind vielmehr bis zum Grunde gur durchlüftet. Lediglich unmittelbar über dem Schlamm kommt es zu Sauerstoffzehrungen. Der Boden der flachen Gewässer ist sandig und teilweise dicht bewachsen. Die tieferen Becken und Rinnen sind mit schwarzem, eisensulfidreichem Faulschlamm angefüllt. In Abbildung 2 sind die großen Faulschlammbecken in den Rügenschen Bodden eingezeichnet. Der Schwarze Faulschlamm in den Senken des Großen Jasmunder Boddens hat nach Bohrungen von WASMUND (1939) eine Stärke von 3 bis $4 \mathrm{~m}$ und enthält an der Oberfläche $90 \%$ Wasser. Eine eigentliche Grenze gegen das Wasser besteht nach WASMUND nicht. Der oberste Meter ist ein allmählicher Übergang von Wasser zu Schlamm. Das mikroskopische Bild zeigt Feindetritus mit Resten pflanzlicher Zellverbände, tierischen Resten, Diatomeen, Markasit, Pyrit und Sandbeimischungen. Der 
$\mathrm{pH}$-Wert beträgt an der Oberfläche 7,2 bis 8,8 . Der Calciumgehalt ist gering (etwa 0,3 bis $0,7 \% \mathrm{Ca}$ der Trockensubstanz; Trahms 1941). Ausführliche Beschreibungen des Untersuchungsgebietes findet man bei Gessner (1937), Trahms \& Stoll (1938) und Overbeck (1956).

Wie Abbildung 1 zeigt, verringert sich der Salz- und Sulfatgehalt kontinuierlich mit steigender Entfernung von der Ostsee und erreicht in Großen Jasmunder Bodden die niedrigsten Werte. Die "marinen" Bestandteile des Brackwassers, hoher Salz- und Sulfatgehalt, verringern sich also mit steigender Verdünnung des Meerwassers. Aber noch im Großen Jasmunder Bodden übertrifft die Sulfatkonzentration mit mehr als $500 \mathrm{mg} \mathrm{SO}_{4}^{--} / 1$ den Sulfatgehalt des Süßwassers um etwa das $Z$ wanzigfache. Umgekehrt steigt die Menge des Phosphats mit steigender Entfernung vom Meer stark an und erreicht im Großen Jasmunder Bodden mehr als $2 \mathrm{mg}$ Gesamt-Phosphor/1, wobei ein deutlicher Anstieg vom Rassower Strom zum Großen Jasmunder Bodden festzustellen ist.

Die Gesamt-Phosphorwerte erscheinen sehr hoch, ließen sich aber in zahlreichen Einzelproben bestätigen. Im Großen Jasmunder Bodden wurden im September 1957 insgesamt 14 Doppelbestimmungen aus allen Tiefenstufen untersucht; sie liegen ausgezeichnet parallel. Mit gleicher Methodik (OHLE 1938) durchgeführte Gesamt-Phosphorbestimmungen von Oberflächenwasser aus dem Arkona-, Bornholm- und Gotlandbecken ergaben unmeßbar kleine Phosphorwerte. Es liegt also zweifellos kein Verfahrensfehler vor. STANGENBERg (1958) hat in einem Brackwassergebiet ganz ähnlicher Struktur, dem Frischen Haff, Gesamt-Phosphorwerte gleicher Größenordnung gemessen. Bei zukünftigen Untersuchungen ist die Frage des Gesamt-Phosphorwertes besonders zu beachten. Im eutrophen Süßwasser mißt man viel geringere Werte. So erhielten wir bei langjährigen Untersuchungen von stark eutrophiertem Havelwasser im Mittel von mehreren hundert Analysen einen Gesamt-Phosphorwert von 610 $\gamma \mathrm{P} / 1$.

Parallel mit dem starken Anstieg des Phosphorgehalts in den Rügenschen Brackwässern steigt auch der Trophiegrad an und der Große Jasmunder Bodden ist ein euproduktives Gewässer. Auf diesen Zusammenhang zwischen Phosphatangebot und Trophiegrad ist bei den entsprechenden Untersuchungen der Rügenschen Bodden immer wieder hingewiesen worden. Die Aufgabe der vorliegenden Arbeit war es deshalb, die Bedeutung des Sediments für die Phosphoranreicherung im Wasser und damit für die Höhe der Produktion in den Rügenschen Bodden quantitativ zu untersuchen, zumal größere Abwassereinflüsse fehlen und deshalb die Phosphorlieferung aus dem Sediment allein für die Produktionshöhe maßgeblich sein mußte. Stickstoff liegt im Wasser der Rügenschen Bodden vorwiegend als Nitrat vor. Die Werte liegen im Bereich von 40 bis 100 y $\mathrm{NO}_{3} / 1$. Ein völliger Verbrauch wurde nie beobachtet. Nitrit und Ammoniak ließen sich höchstens in Spuren nachweisen. Schwefelwasserstoff trat nirgends frei im Wasser auf. Anscheinend wird er nach Freisetzung aus dem sulfidreichen Sediment sofort oxydiert (OVERBEcK 1956). 


\section{DIE SEDIMENTANALYSEN}

\section{Glübverlust, Eisen- und Phospborgehalt der Sedimente}

Einen zusammenfassenden Uberblick über den Glühverlust und Eisen- und Phosphorgehalt der Sedimente gibt Abbildung 2. Der Glühverlust ist im sandigen Sediment des Libben am geringsten, jenem zwischen Hiddensee und Rügen gelegenen,

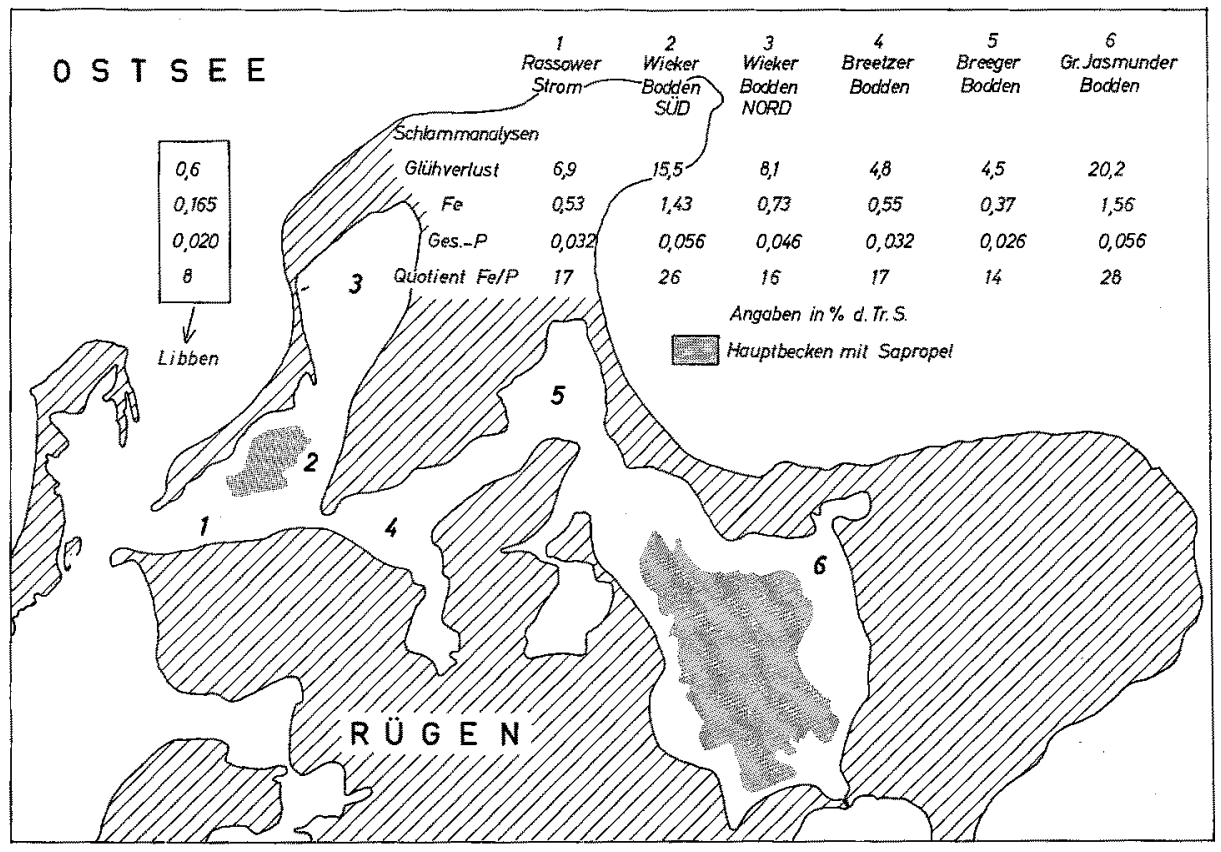

Abb. 2: Untersuchungsgebiet mit den Faulschlammbecken im Großen Jasmunder Bodden (6) und Wieker Bodden Süd (2). Die rechts oben aufgeführten Daten repräsentieren Mittelwerte der Sedimentanalysen (74 Profile mit 295 Zentimeter-Intervall-Proben)

zur eigentlichen Ostsee überleitenden Meeresteil. Er beträgt hier nur 0,6\% der Trokkensubstanz. In allen flachen Bodden mit sandreichem Sediment beträgt der Glühverlust, wie die rechts oben in Abbildung 2 aufgeführten Werte zeigen, recht einheitlich etwa $5 \%$. In den tieferen Faulschlammbecken des Großen Jasmunder Boddens und Wieker Boddens (Süd) ist dagegen erheblich mehr organische Substanz angereichert. Der Glühverlust beträgt hier im Mittel 20,2 bzw. 15,5\% der Trockensubstanz. Der höchste gemessene Wert im Großen Jasmunder Bodden erreichte sogar 27,4\%. Auf Grund ihres Glïhverlustes lassen sich unsere Sedimente zwanglos in zwei Gruppen teilen, in Gyttjen, deren Glühverlust etwa 10 bis $25 \%$ der Trockensubstanz beträgt (UNGEMACH 1960) und in Mineralsedimente mit weniger als $10 \%$ Glihverlust.

Auch bezüglich des Eisen- und Phosphorgehalts lassen sich zwei Gruppen von Sedimenten unterscheiden, die eng mit dem Glühverlust verbunden sind. Denn die Bodden mit dem geringeren Glühverlust haben den niedrigsten Eisen- und Phosphor- 
gehalt. Ebenso wie beim Glühverlust liegen die Werte wieder sehr einheitlich und betragen für Eisen im Mittel 0,54\% der Trodkensubstanz, für Phosphor 0,034\% (Abb. 2). Auch die Eisen/Phosphor-Quotienten dieser Sedimente gleichen sich sehr und liegen recht einheitlich bei 16. Die tieferen Becken bilden erneut eine in sich geschlossene Gruppe. Denn mit steigendem Gluihverlust steigt auch die Eisen- und Phosphormenge im Sediment an, wobei sich der Fe/P-Quotient zugunsten des Eisens verschiebt. Tabelle 1 gibt einen Überblick über diese Verhältnisse. Hier wurden der Eisen- und

Tabelle 1

Glühverlust, Eisen- und Gesamtphosphorgehalt sowie der Eisen/Phosphor-Quotient in den Rïgenschen Bodden und im Großen Plöner See. Ein Vergleich der beiden Sedimenttypen des Rügenschen Brackwassers mit einem Süßwassersediment (Gyttja aus dem Gr. Plöner See, Werte aus UNGEMACH 1960)

\begin{tabular}{|lccc|}
\hline & \multicolumn{2}{c|}{ Rügensche Bodden } & Gr. Plöner See \\
\hline Glühverlust & $<5$ & $>5$ & 19 \\
Eisen (Fe) & 0,3 & 1,2 & 1,1 \\
Ges.-Phosphor $(\mathrm{P})$ & 0,023 & 0,051 & 0,11 \\
Quotient FelP & 12 & 23 & 10 \\
\hline
\end{tabular}

Phosphorgehalt und der Fe/P-Quotient der beiden Sedimentgruppen zusammengestellt. Man sieht, daß bei Sedimenten mit weniger als $5 \%$ Glühverlust der Eisen- und Phosphorgehalt gering ist und der Fe/P-Quotient bei 12 liegt. Mit steigender Menge an organischer Substanz steigt auch die Eisen- und Phosphormenge an, wobei die Eisenmenge den vierfachen, die Phosphormenge jedoch nur den doppelten Wert erreicht. Hierdurch verschiebt sich der Fe/P-Quotient zugunsten des Eisens im Mittel auf 23. Die Erhöhung des Fe/P-Quotienten mit steigendem Glühverlust ist statistisch gut gesichert. Es besteht eine echte Korrelation $(\mathrm{r}=0,85)$ zwischen Glühverlust und $\mathrm{Fe} / \mathrm{P}$-Quotient; wenn der Glihverlust steigt, steigt auch der Fe/P-Quotient. Ein Vergleich der absoluten Eisen- und Phosphormengen mit entsprechenden Analysen anderer Sedimente zeigt, daß der Phosphorgehalt des Brackwasserschlammes sehr niedrig und der Eisengehalt durchaus nicht erhöht ist. So beträgt beispielsweise bei einem Glühverlust von 19\% der Trockensubstanz der Eisengehalt im Nordbecken des Großen Plöner Sees $1,1 \%$, der Phosphorgehalt $0,11 \%$ und der Fe/P-Quotient dementsprechend 10 (vgl. Tab. 1). Der Fe-P-Quotient des Brackwassersediments ist also nicht durch eine Eisenanreicherung, sondern vielmehr durch eine Phosphorabgabe so hoch gestiegen. Nur in den hochproduktiven Abschnitten des Brackwassers ist der Quotient, wie unsere Untersuchungen zeigen, so extrem verschoben. Wir erinnern daran, daß gerade in diesen hochproduktiven Abschnitten des Rügenschen Brackwassers in Ubereinstimmung mit dem hohen Fe-P-Quotienten, also der starken Phosphorabgabe aus dem Schlamm, so extrem hohe Gesamt-Phosphormengen bestimmt wurden. Ein hoher Fe/P-Quotient, starke Phosphatmobilität und hohe Produktion sind also ursächlich miteinander verbunden. Der Fe/P-Quotient kann daher direkt als Indikator für eine Hochproduktion im Brackwasser angesehen werden.

Wie ist die Korrelation zwischen Glühverlust und Fe/P-Quotient zu erklären? Offensichtlich sind eine Reihe von Prozessen verknüpft, die letztlich zu einer Verschie- 
bung des Fe/P-Quotienten führen: Je höher der Glühverlust, also je größer die Menge der organischen Substanz, desto größer ist die Bakterienzahl im Sediment. So ist beispielsweise aus den Kurven von Mechtieva (1963) eine nahezu lineare Beziehung zwischen Bakterienzahl und abbaufähiger organischer Substanz zu ersehen. Je höher die Bakterienzahl im $\mathrm{O}_{2}$-freien Sediment ist, desto intensiver verlaufen die anaeroben Stoffwechselprozesse einschließlich der Bildung von Schwefelwasserstoff. In Zusammenhang mit reduktiven Prozessen sinkt das Redoxpotential ständig ab. Dieser Vorgang läuft bei natürlicher Pufferung des Substrats soweit, daß die Schlammoberfläche, selbst bei Kontakt mit $\mathrm{O}_{2}$-haltigem Wasser, nicht mehr oxydiert wird, die reduktiven Prozesse sich vielmehr bis in das Wasser hinein ausdehnen. Deshalb kommt es zu einer

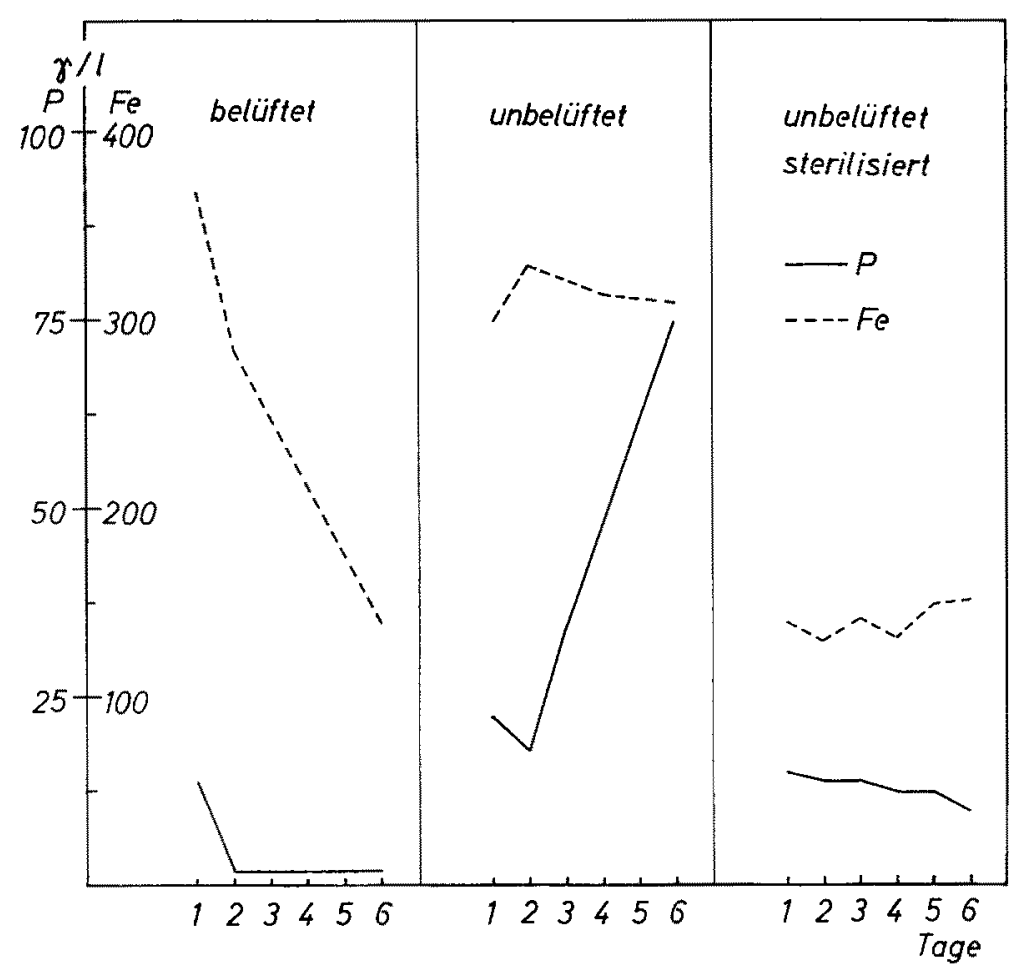

Abb. 3: Ergebnis eines Flaschenversuchs mit Schlamm aus dem Großen Jasmunder Bodden. Der Schlamm wurde frisch verwendet oder zweimal autoklaviert bei $121^{\circ} \mathrm{C}$

weitgehenden Reduktion des Eisens und Festlegung als Sulfid, verbunden mit der Lösung des Phosphats aus der Eisenbindung, was dann zu der geschilderten Erhöhung des $\mathrm{Fe} / \mathrm{P}$-Quotienten führt. Voraussetzung für den ungestörten Ablauf dieser Prozesse ist der hohe Sulfatgehalt des Brackwassers. Ohne ihn wäre eine Abhängigkeit des $\mathrm{Fe} / \mathrm{P}-\mathrm{Quotienten}$ vom Glühverlust nicht möglich, wie wir am Beispiel der Süßwassersedimente noch zeigen werden.

Bei der Verschiebung des Fe/P-Quotienten handelt es sich also offensichtlich um einen mikrobiologischen Prozeß, dessen Intensität von der Menge der organischen 
Substanz im Sediment abhängt. Die große Bedeutung der Mikroflora ließ sich auch in einem Flaschenversuch unter Verwendung von nichtsterilisiertem bzw. sterilisiertem Sediment demonstrieren. Hierzu wurde in einer Flasche zweimal autoklaviertes Sediment aus dem Großen Jasmunder Bodden und ebenso behandeltes Wasser vom gleichen Standort steril angesetzt. In zwei weiteren Flaschen kam nichtsterilisiertes Sediment zur Verwendung. Das Ergebnis des Versuches zeigt Abbildung 3. Man sieht, daß in der belüfteten, nichtsterilisierten Versuchsanordnung der Eisen- und gleichzeitig auch der Phosphorgehalt stark absinken: Das oxydierte dreiwertige Eisen hat den Phosphor mit zu Boden gerissen. Es kommt zu keiner Phosphorabgabe aus dem Sediment. In der unbelüfteten, nichtsterilisierten, bis zum Stopfen mit Wasser gefüllten Flasche gibt der Schlamm dagegen große Mengen von Phosphor an das überstehende Wasser ab. Denn infolge starker Reduktionen wird Phosphor aus der Bindung an das Eisen entlassen und ist als gelöstes anorganisches Phosphat im Wasser nachzuweisen. Ganz andere Verhältnisse liegen dagegen in der Flasche mit sterilisiertem Sediment vor. Obwohl ebenfalls nicht belüftet wurde, wird kein Phosphor aus dem Schlamm abgegeben. Denn ohne die Tätigkeit von Mikroorganismen wird kein Schwefelwasserstoff gebildet, das Eisen damit nicht als Sulfid festgelegt und Phosphor nicht aus der Bindung an das Eisen freigesetzt.

Wir stimmen hinsichtlich dieser Interpretation der Vorgänge im Brackwassersediment völlig überein mit den Ergebnissen OHuEs (1955), der eine nahezu lineare Beziehung zwischen der Sulfatkonzentration und der Schwefelwasserstoffproduktion experimentell bei Konstanthaltung der übrigen Faktoren ermittelte. OHLE konnte in experimentellen Untersuchungen außerdem zeigen, daß die Menge des produzierten Schwefelwasserstoffs außer von der Sulfatkonzentration von der Menge der für die Bakterien zur Verfügung stehenden organischen Substanz bestimmt wird.

\section{Die vertikale Verteilung von Eisen und Phosphor in den Sedimenten}

Die vertikale Verteilung von Eisen und Phosphor in den Sedimenten bietet keine Uberraschungen, denn der Eisen- und Phosphorgehalt bleibt in den 5 bis $10 \mathrm{~cm}$ langen Profilen etwa konstant, was sich auch in den gleichbleibenden Fe/P-Quotienten ausdrückt. Die Korrelation zwischen Glühverlust und Fe/P-Quotient ist deutlich. Gelegentlich, aber durchaus nicht regelmäßig, ist der Fe/P-Quotient an der Sedimentoberfläche erhöht, etwa beim Rassower Strom im September 1954 und beim Wieker Bodden Süd im Oktober 1955. In beiden Fällen liegen Glühverlust und Eisengehalt direkt an der Sedimentoberfläche sehr hoch bei nur wenig erhöhtem Phosphorgehalt, wodurch sich der $\mathrm{Fe} / \mathrm{P}$-Quotient zugunsten des Eisens verschiebt. Wahrscheinlich kam es in beiden Fällen in Zusammenhang mit der starken Erhöhung der organischen Substanz zu einer erhöhten Festlegung des Eisens als Sulfid an der Sedimentoberfläche unter Freisetzung von Phosphor. Gelegentlich wurden auch unregelmäßige Schwankungen des $\mathrm{Fe} / \mathrm{P}$-Quotienten in der Sedimentsäule festgestellt, meist bedingt durch herausfallende Eisen- oder Phosphorwerte, die vielleicht nicht real sind. Herausfallende Werte wurden auch im Breeger Bodden im Mai 1954 beobachtet. Hier wurden in einem Profil trotz des niedrigen Glühverlustes $(2,1 \%)$ hohe Fe/P-Quotienten ermittelt. Aber 
dies alles sind nur Ausnahmen der gesicherten Regel, daß der Fe/P-Quotient mit der Höhe des Glühverlustes parallel läuft.

\section{Der Stickstoffgehalt und der N/P-Qwotient der Sedimente}

Nach Ungemach (1960) enthalten die typischen Gyttjen 0,55 bis 1,0\% GesamtStickstoff der Trockensubstanz, während die Mineralsedimente weniger als 0,5 bis $0,020 \%$ Gesamt-N der Trockensubstanz enthalten. Unsere Werte liegen, wie Tabelle 2

Tabelle 2

Glühverlust, Wasser- und Stickstoffgehalt sowie die Quotienten N/P und Organische Sub$\operatorname{stanz} / P$ der Rügenschen Brackwassersedimente. Angaben in \% der Trockensubstanz; Wasser in $\%$ der Frischsubstanz

\begin{tabular}{|lcccccc|}
\hline & $\begin{array}{c}\text { Rassower } \\
\text { Strom }\end{array}$ & $\begin{array}{c}\text { Wieker } \\
\text { Bodden } \\
\text { Süd }\end{array}$ & $\begin{array}{c}\text { Wieker } \\
\text { Bodden } \\
\text { Nord }\end{array}$ & $\begin{array}{c}\text { Breetzer } \\
\text { Bodden }\end{array}$ & $\begin{array}{c}\text { Breeger } \\
\text { Bodden }\end{array}$ & $\begin{array}{c}\text { Großer } \\
\text { Jasmunder } \\
\text { Bodden }\end{array}$ \\
\hline Glühverlust & 6,9 & 15,5 & 8,1 & 4,8 & 4,5 & 20,2 \\
Wassergehalt & 28 & 45 & 42 & 37 & 31 & 78 \\
Gesamt N & 0,06 & 0,30 & 0,23 & 0,16 & 0,08 & 0,80 \\
N/P & 2,4 & 4,1 & 4,1 & 5,3 & 4,1 & 14,6 \\
Organ. Substanz/P & 216 & 277 & 176 & 104 & 173 & 361 \\
\hline
\end{tabular}

zeigt, genau in diesem Bereich: Die mehr mineralischen Sedimente haben einen Stickstoffgehalt von weniger als $0,5 \%$, der Große Jasmunder Bodden enthält mehr als $0,5 \%$ Gesamt-N der Trockensubstanz. Hierbei besteht eine vollständige Parallelität zwischen Gluhverlust - also organischer Substanz - und Gesamt-Stickstoff, die statistisch sehr gut gesichert ist $(r=0,9)$ und die mit den vorliegenden Ergebnissen an zahlreichen Sedimenten gut ubereinstimmt.

Der N/P-Quotient schwankt $z$ wischen 2,4 und 14,6 bei einem Mittelwert von 4,9 (Tab. 2). Er stimmt damit mit den in der Literatur vorliegenden Werten gut überein. Die Höhe des N/P-Quotienten ist deutlich vom Glühverlust abhängig mit einer gesicherten Korrelation zwischen beiden $(r=0,5)$. Der Quotient steigt also mit steigendem Gluhverlust an: Es ist relativ mehr Stickstoff in den an organischen Stoffen reicheren Sedimenten vorhanden. Diese Verschiebung des N/P-Quotienten dürfte letztlich auf die gleichen Vorgänge zurïckzuführen sein, die auch die Verschiebung des $\mathrm{Fe} / \mathrm{P}$ Quotienten bewirkten, nämlich auf die verstärkte Freisetzung von Phosphor aus Sedimenten mit reicherem organischem Anteil, wodurch sich der Anteil des Stickstoffs im Verhältnis zum Phosphor relativ erhöht. Das gleiche drückt sich auch im Quotienten Organische Substanz (Glübverlust) : $P$ aus (Tab. 2), der parallel zum Fe/P-Quotienten und N/P-Quotienten ebenfalls im Großen Jasmunder Bodden den bei weitem höchsten Wert erreicht. An zweiter Stelle steht wie beim Fe/P-Quotienten wiederum der Wieker Bodden Süd.

In der vertikalen Verteilung des Gesamt-Stickstoffs im Sediment ist, soweit unsere Daten eine Aussage zulassen, eine Abnahme des Stickstoffs mit zunehmender Sedimenttiefe festzustellen. 


\section{Der Wassergebalt der Sedimente}

Wie aus Tabelle 2 ersichtlich, besteht eine deutliche Parallelität zwischen Glühverlust und Wassergehalt der Sedimente. Der Große Jasmunder Bodden hat bei größtem Glühverlust auch den höchsten Wassergehalt. Der höchste Mittelwert für eine Sedimentsäule beträgt 87,5\% Wasser (Großer Jasmunder Bodden), der absolut höchste Wert im gleichen Bodden 91,1\%, der absolut niedrigste $5 \%$ (Rassower Strom). Aber auch im Rassower Strom steigt der Wassergehalt sofort an, wenn der Glühverlust der Sedimente ansteigt (April 1955: Mittelwert Glühverlust 15,5\%, Wassergehalt 76,5\%). Die Korrelation zwischen Glühverlust und Wassergehalt ist statistisch gesichert $(r=0,6)$. Vielfach ist die oberste Sedimentschicht am wasserreichsten (in $57 \%$ aller Profile). Häufig ist ein Sprung im Wassergehalt vom ersten zum zweiten Zentimeter zu beobachten, der teilweise sehr erheblich sein kann (beispielsweise von $75,4 \%$ auf $47,6 \%$. Auch UNGEMACH weist auf die ausschlaggebende Bedeutung der organischen Substanz für den Wassergehalt der Sedimente hin, die auf Grund ihres Quellungsvermögens eine hohe Wasserkapazität besitzt. Bei Fehlen dieses Quellkörpers in den mineralischen Sedimenten nimmt der Wassergehalt sofort stark ab.

Ein durchschnittlicher Wassergehalt von $78 \%$, wie er im Großen Jasmunder Bodden gefunden wurde, entspricht einem Sediment vom Gyttja-Typ; ein Wassergehalt von 20 bis $40 \%$ wird für mineralische Sedimente angegeben (UNGEMACH 1960). Unsere Sedimente lassen sich also auch nach dem Wassergehalt - ebenso wie nach dem Glühverlust, dem Eisen- und Phosphorgehalt und dem Fe/P-Quotienten - in zwei Gruppen gliedern, was auf die ursächliche Verknüpfung dieser Faktoren hinweist.

\section{BESPRECHUNG DER ERGEBNISSE}

Die untersuchten Sedimente vom Gyttja-Typ des Rügenschen Brackwassers enthalten etwa 1 bis $2 \%$ Eisen und etwa 0,05\% Phosphor der Trockensubstanz. Es konnte festgestellt werden, daß sich der Fe/P-Quotient gesetzmäßig mit Erhöhung des Glühverlustes zugunsten des Eisens verschiebt. So sind die an den organischen Stoffen reicheren Sedimente durch einen hohen Fe/P-Quotienten gekennzeichnet. Die Verschiebung des $\mathrm{Fe} / \mathrm{P}-\mathrm{Quotienten}$ ist statistisch signifikant. Die Verschiebung des Fe/P-Quotienten ist im wesentlichen durch die im sulfatreichen Brackwasser erhöhte Festlegung des Eisens als Sulfid und die damit verbundene Freisetzung des Phosphors aus dem Sediment bedingt. Diese Verhältnisse gelten zunächst nur für die untersuchten Rügenschen Brackwässer. Es ist anzunehmen, daß in ähnlichen Brackwässern grundsätzlich gleiche Abläufe auftreten werden. Jedoch könnten allochthone Einflüsse, besonders die Zufuhr von Eisen und Huminsäuren, den Fe/P-Quotienten stark beeinflussen. In solchen Fällen dürfte dann die für die Rügenschen Brackwässer gültige Korrelation zwischen Glühverlust und Fe/P-Quotient gestört sein.

Nach den Analysen von StANgEnberg (1958) aus dem Frischen Haff errechnet sich für das Sediment dieses Brackwassers ein Fe/P-Quotient von 15 bis 19. UNGEMACH (1960) hat bei seinen Untersuchungen des Sedimentchemismus von 40 europäischen Seen auch zwei Brackwässer mit einbezogen, den Waterneverstorfer Binnensee und das 
Windebyer Noor, die beide -- wenn auch durch Schleusen abgetrennt - mit der Ostsee in Verbindung stehen und deren Chemismus, soweit für unsere Betrachtungen wichtig, zusammen mit den Werten des Großen Jasmunder Boddens in Tabelle 3 wiedergegeben

\section{Tabelle 3}

Chemismus dreier Brackgewässer. Angaben in \% der Trockensubstanz (Wasser in \% der Frischsubstanz). Waterneverstorfer Binnensee und Windebyer Noor nach Werten von UNGEMACH (1960); Großer Jasmunder Bodden nach eigenen Daten

\begin{tabular}{|c|c|c|c|}
\hline & $\begin{array}{c}\text { Waterneverstorfer } \\
\text { Binnensee }\end{array}$ & $\begin{array}{l}\text { Windebyer } \\
\text { Noor }\end{array}$ & $\begin{array}{l}\text { Gr. Jasmunder } \\
\text { Bodden }\end{array}$ \\
\hline \multicolumn{4}{|l|}{ Im Schlamm: } \\
\hline Glühverlust & 22 & 18 & 20 \\
\hline Gesamtstickstoff & 1,4 & 0,8 & 0,8 \\
\hline Gesamtphosphor & 0,12 & 0,087 & 0,056 \\
\hline Eisen & 1,80 & 1,90 & 1,56 \\
\hline Wasser & 84 & 80 & 78 \\
\hline Felp-Quotient & 15 & 22 & 28 \\
\hline N/P-Quotient & 12 & 9 & 14 \\
\hline \multicolumn{4}{|l|}{ Im Wasser: } \\
\hline $\mathrm{SO}_{4}^{--} \mathrm{mg} / \mathrm{l}$ & 52 & 370 & 530 \\
\hline $\mathrm{NaCl} \%$ & 3,33 & 4,18 & 6,94 \\
\hline
\end{tabular}

ist. Das Windebyer Noor entspricht weitgehend dem Großen Jasmunder Bodden mit dem Unterschied, daß der Rügensche Bodden einen höheren Sulfat- und Salzgehalt besitzt. Sein Phosphorgehalt ist etwas niedriger, dementsprechend steigt der Fe/P-Quotient des Großen Jasmunder Boddens über den Wert des Windebyer Noors. Anders dagegen der Waterneverstorfer Binnensee, der bei gleichen Eisenwerten einen erheblich höheren Phosphorgehalt und einen dementsprechend niedrigeren Fe/P-Quotienten besitzt. Der Sulfatgehalt beträgt hier nur $1 / 10$ des Wertes vom Großen Jasmunder Bodden. Man ist versucht, die Unterschiede im Phosphatgehalt der drei Sedimente und damit auch der Fe/P-Quotienten direkt in Abhängigkeit vom abgestuften Sulfatgehalt zu sehen, also eine Korrelation zwischen Sulfatgehalt und Fe/P-Quotienten anzunehmen. Hiermit würde dann noch einmal mit aller Deutlichkeit die Bedeutung des Sulfats für den Stoffhaushalt, speziell für die Phosphatfreisetzung aus dem Sediment, unterstrichen. Innerhalb eines Gewässersystems mit etwa gleichhohem Sulfatgehalt, wie es die Rügenschen Brackwässer darstellen, ist dann der Gehalt an organischer Substanz des Sediments für den Fe/P-Quotienten, also für die Abgabe von Phosphor aus dem Sedi-ment, verantwortlich, wie aus der gesicherten Korrelation zwischen organischer Substanz und Fe/P-Quotient hervorgeht.

Wie wirksam dieser Mechanismus der Phosphorabgabe sein muß, zeigt folgende Uberlegung: Die sehr hohe Planktonproduktion im Großen Jasmunder Bodden beruht auf einer riesigen Blaualgen- und Nannoplanktonentwicklung, die mehrere Millionen Zellen im Milliliter betragen kann (Overbeck 1962). Dementsprechend ist die Sedimentationsrate sehr hoch und beträgt nach Angaben von WASMUND (1939) jährlich 3 bis $4 \mathrm{~cm}$. Bei dieser Sedimentationsrate hätte man eine Phosphoranreicherung an der Sedimentoberfläche erwarten müssen. Jedoch das Gegenteil ist der Fall: Das Sediment ist an Phosphor verarmt. Es muß sich also ein Gleichgewicht zwischen Sedimentation, 
Mineralisierung und Freisetzung von Phosphor eingestellt haben, das den Phosphorgehalt des Schlammes auf so niedriger Höhe hält. Der hohe Anteil von wasserlöslichem Phosphat im Sediment gibt einen Begriff von der Phosphatmobilisierung.

Das bisher Ausgeführte galt ausschließlich für das Brackwasser. Zur Prüfung der Verhältnisse im Süßwassersediment berechneten wir aus den Analysendaten von UNGEMACH (1960) für die Seen mit Gyttjen die Korrelation zwischen Glühverlust und Fe/P-Quotient, Fe/P-Quotient und Gesamt-Schwefel sowie Fe/P-Quotient und Sulfidgehalt der Sedimente. Es konnte in keinem Falle eine gesicherte Korrelation ermittelt werden. Auch UnGEMACH (1960) mißt dem Fe/P-Quotienten des Süßwassersediments keine indikatorische Bedeutung bei. Der Quotient verändert sich vielmehr unabhängig vom Glühverlust in unïbersichtlicher Weise. Das gleiche Ergebnis brachten auch Berechnungen an Mineralsedimenten des Süßwassers. Damit kommen wir zu einem wichtigen Unterschied zwischen Süßwasser- und Brackwassersediment: Die Schwefelwasserstoffbzw. Eisensulfidbildung ist, wie oben ausgeführt, von zwei Faktoren abhängig, Sulfatangebot und abbaufähiger organischer Substanz. $\mathrm{Da}$ im Brackwasser stets genügend Sulfat vorhanden ist, hängt hier die Sulfidbildung allein von der Menge der organischen Substanz ab. Die organische Substanz des Sedimentes, deren Menge von der Planktonproduktion bedingt ist, bestimmt damit die Größe des Fe/P-Quotienten. Der Fe/PQuotient ist somit zu einem produktionsbiologischen Indikator des Brackwassers geworden. Im Gegensatz hierzu fehlt im Süßwasser der dominierende Sulfat-Faktor, wodurch es zu unregelmäßigen und unübersichtlichen Verschiebungen des Fe/P-Quotienten kommt.

Wir verstehen jetzt das Wesen der außerordentlich hohen Produktion im Brackwasser: Weitgehende Phosphatmobilisierung hält die Produktion stets auf großer Höhe. Die Nutznießer des hohen Phosphatangebots und damit die wichtigsten Produzenten sind neben Blaualgen die Phytoplankter vom Nannoplankton-Typ. Mobilisiertes Phosphat und Nannoplankton, das sind die beiden Säulen der riesigen Produktion des Rügenschen Brackwassers. Für die Beurteilung der jeweiligen Situation bietet sich der $\mathrm{Fe} / \mathrm{P}$-Quotient als nützlicher Indikator at.

Ausführliche Analysentabellen können beim Verfasser angefordert werden.

\section{ZUSAMMENFASSUNG}

1. Zur Untersuchung des Phosphathaushalts der Rügenschen Brackwässer (Ostsee) wurden zahlreiche Sedimentanalysen durchgeführt, und dabei Wassergehalt, Glühverlust, Eisen, Phosphor und Stickstoff untersucht. Die bis $10 \mathrm{~cm}$ langen Sedimentprofile wurden hierzu in $1 \mathrm{~cm}$ lange Abschnitte zerlegt.

2. In methodischen Untersuchungen wurde festgestellt, daß ein Salzgehalt von $1 \%$ $\mathrm{NaCl}$ die Phosphatbestimmung nicht beeinflußt. Erst ab $3 \% \mathrm{NaCl}$ wurde ein deutlicher Salzfehler festgestellt. Zwei miteinander verglichene Verfahren der GesamtPhosphorbestimmung (Schwefelsäure- und Salzsäureaufschluß) ergaben eine befriedigende Übereinstimmung.

3. In den Rügenschen Brackwässern steigt der Phosphorgehalt mit steigender Entfernung von der Ostsee stark an und erreicht im Gesamt-Phosphat Werte von mehr als $2 \mathrm{mg} P / 1$. Gleichzeitig steigt auch die Produktion stark an. 
4. Organische Substanz (Glühverlust), Eisen und Phosphor des Sediments sind eng miteinander verknüpt: Bei Sedimenten mit weniger als $5 \%$ Glühverlust ist der Eisen- und Phosphorgehalt gering, der Fe/P-Quotient liegt bei 12. Mit steigender Menge an organischer Substanz steigt der Eisen- und Phosphorgehalt des Sediments an, wobei Eisen den vierfachen, Phosphor aber nur den doppelten Wert erreicht. Hierdurch verschiebt sich der Fe/P-Quotient zugunsten des Eisens im Mittel auf 23. Da der Eisengehalt im Vergleich zu Süßwassersedimenten mit gleichem Glühverlust nicht erhöht ist, der Phosphorgehalt dagegen sehr niedrig liegt, ist der Fe/P-Quotient nicht durch eine Eisenanreicherung im Schlamm, sondern vielmehr durch eine Phosphorabgabe aus dem Schlamm so stark verschoben.

5. Voraussetzung für diese Prozesse im Brackwassersediment ist der hohe Sulfatgehalt des Brackwassers und die damit verknüpte hohe Sulfatreduktion, die zur Eisensulfidbildung und damit zur Lösung der adsorptiven Bindung zwischen Ferrihydroxyd und Phosphat führt. Die aus der Bindung befreiten Phosphat-Ionen gehen wieder in Lösung, während Eisen als Sulfid im Sediment festgelegt wird. Der Sulfatgehalt ist also für den Phosphathaushalt von entscheidender Bedeutung.

6. Im Sediment des sulfatärmeren Süßwassers fehlt die Korrelation zwischen organischer Substanz und Fe/P-Quotient. Der Fe/P-Quotient verändert sich hier vielmehr unabhängig vom Glühverlust. Die Korrelation zwischen Fe/P-Quotient und Glühverlust ist also vom Sulfatgehalt abhängig.

7. Nur in den hochproduktiven Abschnitten des Brackwassers ist der Fe/P-Quotient so extrem verschoben. Ein hoher Fe/P-Quotient, starke Phosphatmobilisierung und hohe Produktion sind also ursächlich miteinander verknüpt. Der Fe/P-Quotient kann daher direkt als Indikator fïr eine Hochproduktion im Brackwasser angesehen werden.

8. Auch der N/P-Quotient und der Wassergehalt des Brackwassersediments sind mit dem Glühverlust verbunden und steigen mit dem Glühverlust an.

9. Zwei Hauptprinzipien sind für die riesige Produktion in den Rügenschen Brackwässern verantwortlich: die hohe Phosphorabgabe aus dem Schlamm und die bereits früher festgestellte gewaltige Nannoplanktonentwicklung.

Die Untersuchungen wurden an der Biologischen Forschungsanstalt Hiddensee der Universität Greifswald und dem Botanischen Institut Potsdam-Sanssouci durchgeführt und an der Hydrobiologischen Anstalt der Max-Planck-Gesellschaft zum Abschluß gebracht. Frau Gertrud NotNi sei für ihre unermüdliche Hilfe herzlich gedankt. Sehr zu Dank verpflichtet bin ich auch Frau Dr. Ragna Rathsack-KünZenbach für ihre unentbehrliche Hilfe bei der Probenentnahme. Herrn Prof. Dr. W. OHLE danke ich für die kritische Durchsicht des Manuskriptes und wertvolle Anregungen.

\section{ZITIERTE LITERATUR}

Cooper, L. N. H., 1938/39. Salt error in determinations of phosphate in sea water. $J$. mar. biol. Ass. U.K. 23, 171-178.

EINSELE, W., 1936. Ưber die Beziehungen des Eisenkreislaufs zum Phosphatkreislauf im eutrophen See. Arch. Hydrobiol. 29, 664-686.

GessNer, F., 1937. Hydrographie und Hydrobiologie der Brackwässer Rügens und des Daris. Kieler Meeresforsch. 2, 1-80. 
Kalle, K., 1935. Meereskundliche chemische Untersuchungen mit Hilfe des Zeiß'schen PulfrichPhotometers. IV. Mittl. Der Einfluß chemischer Stoffe auf die Phosphatgehaltsbestimmung. Annln Hydrogr. Berl. 63, 58-65.

Krause, H. R., 1961. Einige Bemerkungen über den postmortalen Abbau von SüßwasserZooplankton unter Laboratoriums- und Freilandbedingungen. Arch. Hydrobiol. 57, $539-543$.

Mechtieva, W. L., 1963. Microflora distribution in modern deposits of the Great Sevan Lake. (Russ. mit engl. Zusammenfsg.) Mikrobiol., Moskva 32, 113-120.

Mǘlez, G.-O., 1954. Praktikum der quantitativen chemischen Analyse. 3. Aufl. Hirzel, Leipzig, $407 \mathrm{pp}$.

OHLE, W., 1935. Organische Kolloide in ihrer Wirkung auf den Stoffhaushalt der Gewässer. Naturreissenschaflen 23, 480-484.

- 1937. Kolloidgele als Nährstoffregulatoren der Gewässer. Naturceissenschaften 25, 471-474.

- 1938. Zur Vervollkommnung der hydrochemischen Analyse. III. Die Phosphorbestimmung. $Z$. angew. Chem. 51, 906-911.

- 1953. Der Vorgang rasanter Seenalterung in Holstein. Naturwissenscbaften 40, 153-162.

- 1954a. Sulfat als „Katalysator" des limnischen Stoffkreislaufes. Vom Wasser 21, 13-32.

- 1954b. Die zivilisatorische Schädigung der holsteinischen Seen. Städtebygiene 9, 1-5.

- 1955. Die Ursachen der rasanten Seeneutrophierung. Verh. int. Ver. Limnol, 12, 373-382.

OVERBECK, J., 1956. Untersuchungen über die Standortverhältnisse an den Wuchsorten von Fucus vesiculosus in den Gewässern von Hiddensee und Rügen. (Fucus-Untersuchungen II.) Arch. Protistenk. 101, 401-442.

- 1957. Ein Bodenstecher zur Probenentnahme in flachen Gewässern. Z. Fisch. N. F. 5, 285-288.

- 1962. Das Nannoplankton ( $\mu$ - Algen) der Rügenschen Brackwässer als Hauptproduzent in Abhängigkeit vom Salzgehalt. Kieler Meeresforsch. 18, 157-171.

StANgenBerg, M., 1958. Chemisch-physikalische Ursachen der ungeheuren Planktonproduktion im Frischen Haff. Verh. int. Ver. Limnol. 13, 400-410.

Thomas, E. A., 1955. Stoffhaushalt und Sedimentation im oligotrophen Aegerisee und im eutrophen Pfäffiker- und Greifensee. Mem. Ist. ital. Idrobiol. (Suppl.) 8, 357-465.

Tranms, O.-K. \& STOLL, K., 1938. Hydrobiologische und hydrochemische Untersuchungen in den Rügenschen Boddengewässern während der Jahre 1936 und 1937. Kieler Meeresforsch. 3, 61-98.

TraHms, O.-K., 1941. Die Bedeutung des Untergrundes für die Nährstoff regeneration in stark eutrophen Brackgewässern. Z. Fisch. 39, 725-748.

UnGEMACH, H., 1960. Sedimentchemismus und seine Beziehungen zum Stoffhaushalt in 40 europäischen Seen. Diss. (Ms.) Kiel, 420 pp.

Wasmund, C., 1939. Sedimentationsgeschichte des Großen Jasmunder Boddens. Geologie Meere Binnengew. 3, 506-526.

\section{Diskussion im Anschluß an den Vortrag Overbeck}

JARKE: Welcher Abschnitt des Sediments wurde untersucht und den Angaben in den Tabellen zugrunde gelegt?

OVERBEck: Die Bodenstecherproben wurden in $1 \mathrm{~cm}$ lange Abschnitte zerlegt und zentimeterweise untersucht. Die Länge der Profile betrug maximal $12 \mathrm{~cm}$. Die Werte in den Tabellen sind Mittelwerte aus rund 260 Einzelproben.

JARKE: Aus welchen Gründen änderten sich um 1860 - offenbar ziemlich plötzlich - die Sedimentationsverhältnisse; weshalb wurde um diese Zeit die Gyttja-Sedimentation durch eine Sapropelsedimentation abgelöst?

Overbeck: Wie Bohrungen von WASMUND (1939) zeigten, geht etwa ab 1860 (Datierung durch massiertes Auftreten von Kiefernpollen aus Aufforstungen der Zeit) die Gyttja des Großen Jasmunder Boddens in Faulschlamm über. In den letzten hundert Jahren hat sich eine 3 bis 
$4 \mathrm{~m}$ dicke Faulschlammschicht am Boden des Großen Jasmunder Boddens abgesetzt. Für diesen plötzlichen Umschlag zur Faulschlammbildung dürfte eine Reihe von Gründen anzuführen sein: Durch den Bau der Bahnstrecke Stralsund-Saßnitz wurde der Große Jasmunder Bodden vom Kleinen Jasmunder Bodden abgeschnitten, wodurch zweifellos der Durchfluß verringert wurde. Durch Straßenbau und Dünenschutz auf der Schaabe, einer schmalen Nehrung, die den Bodden von der Ostsee abtrennt, wurden Sturmflutdurchbrïche und damit die Zufuhr frischen Salzwassers abgestoppt. Entscheidend dürfte aber sein, daß zu diesem Zeitpunkt (1860) das Gewässer so flach geworden war, daß eine dauernde Durchmischung bis zum Grunde die Regel ist. Hierdurch wird der mineralisierte und aus der Bindung an das Eisen befreite Phosphor sofort wieder der Produktionszone zugeführt und es kommt zu der oben geschilderten außerordentlichen Produktionshöhe, die von einer gleich hohen Sedimentation $(3-4 \mathrm{~cm}$ im Jahr) begleitet ist. Das Uberangebot von organischer Substanz im Sediment führt zur Bildung von Faulschlamm. Nach Angaben von HüBEL (unpublizierte, briefliche Mitt.) erreicht die maximale Produktion im Großen Jasmunder Bodden den riesigen Wert von $5,7 \mathrm{~g} \mathrm{C} / \mathrm{m}^{2} / \Gamma \mathrm{ag}$. Die bisher in der Literatur bekannten maximalen Werte liegen im Bereich von $4 \mathrm{~g} \mathrm{C} / \mathrm{m}^{2} / \mathrm{Tag}$.

OHLE: Die Damm-Abriegelung des Großen vom Kleinen Jasmunder Bodden wird vermutlich die Wasserströnungen verringert und die Fermentation des Schlammes verstärkt haben; die Sulfatreduktion und Freisetzung des an Eisen gebundenen Phosphats infolge Eisensulfidbildung wurden intensiviert. Infolgedessen blieb der Phosphor wie in einem „kurzgeschlossenen Stoff-kreislauf" in Umlauf zwischen pflanzlicher (Plankton-) Inkarnation und Mineralisierung. Schwache P-Verluste durch doch noch eintretende Sedimentation und entsprechende Festlegung im Schlamm werden wie bei anderen Gewässern sicherlich durch Zufuhr aus der BoddenUmgebung und aus der Atmosphäre (durch aeolischen Transport oder Erosionswasser) ausgeglichen. 